\title{
Excitation of the oscillations in roAp stars: Magnetic fields, diffusion, and winds
}

\author{
M.S. Cunha ${ }^{1}$, S. Théado ${ }^{2}$ and S. Vauclair ${ }^{3}$ \\ ${ }^{1}$ Centro de Astrofísica da Universidade do Porto, Rua das Estrelas, 4150-762 Porto, Portugal \\ email: mcunha@astro.up.pt \\ ${ }^{2}$ Institut d'Astrophysique et de Géophysique, Université de Liège, allée du Six Août 17, 4000 \\ Liège, Belgium \\ ${ }^{3}$ Observatoire Midi Pyrénées, 14, Avenue Edouard-Belin, 31400-Toulouse, France
}

\begin{abstract}
We discuss different physical aspects that may influence pulsation stability in rapidly oscillating Ap stars. We pay particular attention to the role of the magnetic field, atomic diffusion and winds and point out some of the important uncertainties involved in present studies of pulsation stability in these stars. We argue that presently the models that best reproduce the observations are those with convection suppressed, diffusion of helium, and no winds. With these models, in the region of the HR diagram where most roAp stars have been found, we predict the excitation of high order modes and the damping of low order modes, in accord with observations.
\end{abstract}

Keywords. Stars: chemically peculiar, stars: magnetic fields, stars: oscillations

\section{Introduction}

Since they were first discovered (Kurtz 1982), rapidly oscillating Ap (roAp) stars have challenged us in many different ways. Perhaps one of the most interesting questions raised soon after they were discovered was, 'why do these stars pulsate in the way they do?'

Even though the exact position of roAp stars in the HR diagram is still under debate, due, particularly, to the lack of good determinations of their effective temperatures, they are generally found in the Main Sequence part of the Classical Instability Strip, similar to the $\delta$-Scuti stars. Thus, nonadiabatic calculations of linear oscillations performed with the original models of roAp stars predicted that their pulsations should have periods of few hours, rather than periods of few minutes, as observed by Don Kurtz. This problem, first discussed by Dolez \& Gough (1982), is not yet fully resolved, despite having attracted the attention of many different investigators.

In this paper we will discuss how different aspects of the physics of roAp stars may play a role in determinng which oscillations are excited. This study follows those of Balmforth et al. (2000) (hereafter BCDGV) and Cunha (2002). A description of the codes used for calculating the equilibrium and pulsating models used throughout this paper can be seen in section 3.3 of BCDGV.

\section{Building nonspherically symmetric models}

Rapidly oscillating Ap stars are known to be patchy. This is mainly a result of the combination of magnetic fields and atomic diffusion. When carrying out theoretical studies of oscillations in these stars, one possible way to deal with the lack of spherical symmetry is to make use of the variational principal. Under given conditions, the variational principle can be used to calculate the frequencies of the oscillations appropriate to models 
composed of different angular regions, through a weighted average of the frequencies appropriate to different spherically symmetric models.

With this in mind, throughout the paper we will study spherically symmetric models only. To learn about the oscillation properties in models composed of different regions, it is enough to combine the correct spherically symmetric models in the way described in section 4 of BCDGV.

\section{Some key ingredients for pulsation stability in roAp stars}

To understand, simultaneously, the presence of high order modes and the absence of low order modes in roAp stars, it is important to focus on the properties that distinguish the latter from the $\delta$-Scuti stars. In particular roAp stars are slow rotators and have strong magnetic fields. Consequently, very efficient diffusion processes, strongly influenced by the magnetic fields, take place in their surface layers. With this in mind, below we list some key ingredients that are likely to influence the pulsation stability in roAp stars.

(i) Indirect effects of the magnetic field - interaction with convection;

(ii) Chemical gradients - diffusion, winds;

(iii) Direct effects of the magnetic field - interaction with pulsation;

(iv) Wave reflection in the surface layers;

(v) Etc.

\subsection{Indirect effects of the magnetic field - interaction with convection}

Strong magnetic fields influence convective motions. In a simple picture, in stars convective motions distort the magnetic field lines, and the Lorentz force resulting from the perturbation of the magnetic field may prevent convection from proceeding as it otherwise would. The interaction between magnetic fields and convection was studied in particular by Gough \& Tayler (1966) and Moss \& Tayler (1969). Moreover, the sufficient condition for suppression of convection derived by Gough \& Tayler was tested by BCDGV for magnetic fields of magnitudes of a few $\mathrm{kG}$, in models appropriate for roAp stars. These studies indicate that magnetic fields like those observed in roAp stars are sufficiently strong to influence envelop convection, and, possibly suppress it to a large extent, particularly in regions where the magnetic field is close to vertical. With this in mind BCDGV studied linear, nonadiabatic oscillations in models of roAp stars with envelop convection suppressed, and found that in these models high order modes, in the frequency range observed in roAp stars, were unstable. Moreover, they found that the excitation of these modes takes place in the region where hydrogen is ionized, while the region of second ionization of helium contributes to stabilize them (as suggested by Dziembowski \& Goode (1996)).

With the goal of expanding the work of BCDGV, Cunha (2002) studied linear nonadiabatic oscillations in models with convection suppressed, spanning the region of the HR diagram where roAp stars are located. Fig. 1 shows the Theoretical Instability Strip (TIS) derived by Cunha (2002), together with those roAp stars for which Hipparcos parallaxes are available. Even though a general agreement was found between the observations and the TIS, a few discrepancies remain to be explained. In particular, a few stars are located beyond the theoretical red edge, the theoretical blue edge appears to be too hot, and, unlike what is observed, low order modes, with frequencies typical of those observed in $\delta$-Scuti stars, are also predicted to be unstable. 


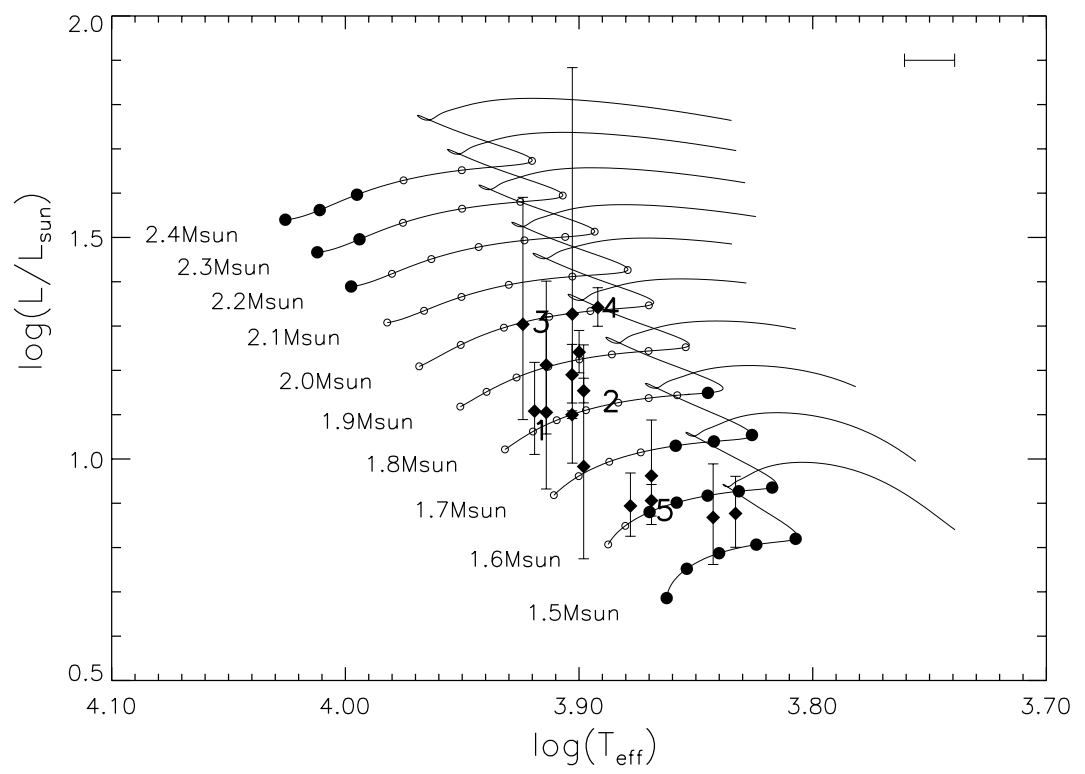

Figure 1. Theoretical Instability Strip (TIS) for roAp stars as derived by Cunha (2002). Modes like those observed in roAp stars are predicted to be unstable in the region filled with small open circles. roAp stars for which Hipparcos parallaxes are available are also shown. The numbers 1 to 5 correspond to models represented in Fig. 2 and Fig. 3

\subsection{Chemical gradients - diffusion, winds}

If the excitation of the oscillations observed in roAp stars depends strongly on nonadiabatic processes associated with the regions where hydrogen and helium are ionized, as suggested by the work of BCDGV, then the profiles of these two elements in the envelopes of roAp stars may play an important role in the excitation.

Theado et al. (in preparation) evolved models of roAp stars from the ZAMS, suppressing convection whenever a simplified criteria for suppression of convection (also tested by BCDGV) was satisfied. Since the intention was to test the influence that chemical profiles appropriate to different physical conditions would have in the excitation process, some of the models which were evolved accounted only for the diffusion of helium while others included the combined effect on helium of atomic diffusion and winds.

When no wind is present, helium simply sinks, and the regions where hydrogen and helium are ionized become very poor in helium and, consequently, rich in hydrogen. On the other hand, when a wind is present to compete with diffusion, an accumulation of helium takes place in the region where helium has its first ionization. Helium profiles were computed for models spanning the TIS of Cunha (2002), and the stability analysis of linear oscillations appropriate to these models was carried out.

In Fig. 2 we show the results of the stability analysis for models with different helium profiles. It is clear from the results that in the region of the HR diagram where these models are located, models with diffusion and no winds tend to show fewer unstable low order modes of the type observed in $\delta$-Scuti stars, while showing unstable high order modes with frequencies typical of those observed in roAp stars. Since these models are located in the region where most roAp stars seem to be concentrated, this might be an indication that the levitation of helium by a wind is not a common phenomenon in roAp stars. The diffusion of helium, on the other hand, might be part of the solution for 


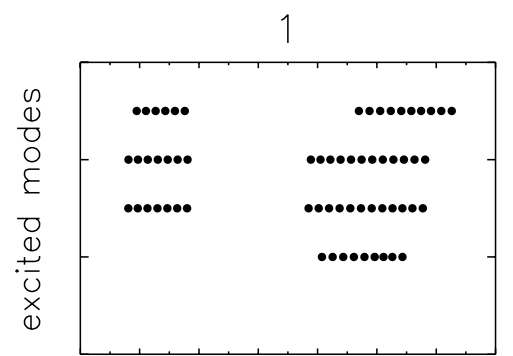

0.00 .51 .01 .52 .02 .53 .03 .5 frequency $(\mathrm{mHz})$

3

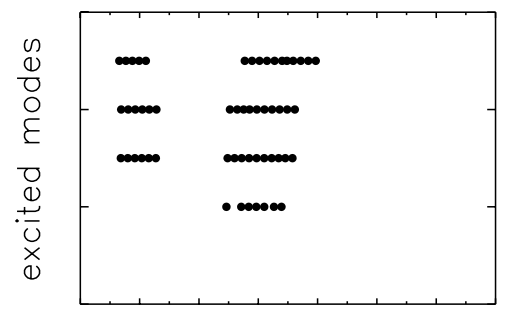

0.00 .51 .01 .52 .02 .53 .03 .5 frequency $(\mathrm{mHz})$

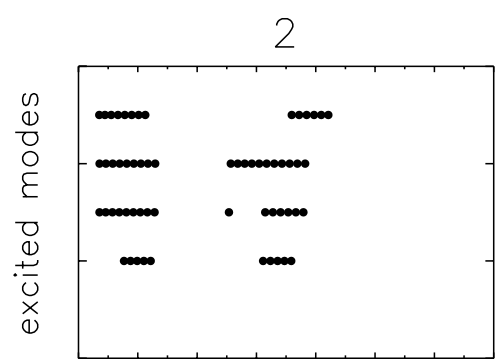

0.00 .51 .01 .52 .02 .53 .03 .5 frequency $(\mathrm{mHz})$

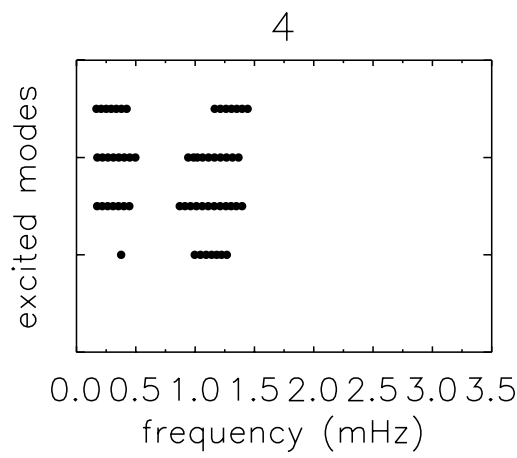

Figure 2. Each panel corresponds to a given location in the TIS (identified by the corresponding number in Fig. 1). In each panel results are given for four different models: homogeneous envelop (top), diffusion of helium plus stronger wind (second from top), diffusion of helium plus weaker wind (third from top) and diffusion of helium only (bottom). Winds were parametrized in terms of the helium outgoing flux, resulting in mass loss rates that vary slightly from model to model. The averages over the four panels are $\approx 5 \times 10^{-14} M_{\odot} \mathrm{yr}^{-1}$ for weaker winds, and $\approx 14 \times 10^{-14} M_{\odot} \mathrm{yr}^{-1}$ for stronger winds. Each filled circle corresponds to the frequency of an unstable mode.

the original mismatch between the predictions of BCDGV and Cunha (2002), and the observations, concerning the excitation of low order modes in roAp stars.

Another mismatch between the TIS and the observations is the position of the theoretical red edge, which appears to be too hot. With this in mind tests with different helium profiles where also carried out for models around the theoretical red edge. However, the position of the theoretical red edge was found to be unchanged, regardless of the helium profile considered.

\section{3. ... etc.}

Still with the problem of the red edge in mind, let us jump to the end of the list given at the start of this section.

The surface layers of roAp stars are rather complicate and often badly modeled. It is, therefore, important to study the sensitivity of the results to those physical aspects that are not yet well known, or not yet well modeled. Following Cunha (1999) and BCDGV, we have explored different $T-\tau$ relations in the atmosphere, different external boundary conditions in the pulsating code, and different extents of the atmosphere. We paid particular attention to models of lower masses and effective temperatures, to check the robustness of the theoretical red edge. 


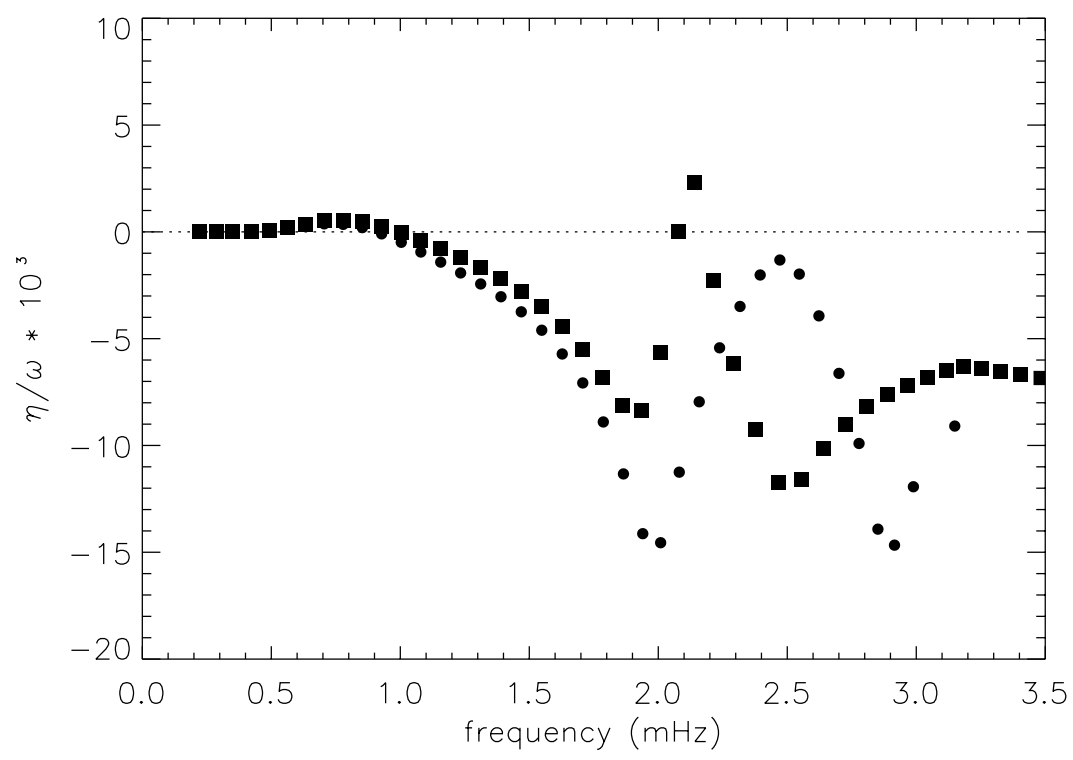

Figure 3. Relative growth rates for a model which location is identified in Fig. 1 by the number 5. The circles and squares show the growth rates for models with diffusion and no wind, and atmospheres which extend to optical depths of $\tau=10^{-4}$ and $\tau=10^{-6}$, respectively. The modes are excited if their growth rates are positive.

Fig. 3 shows the relative growth rates for oscillations in two models of $M=1.6 M_{\odot}$, which atmospheres extend to different optical depths. When the atmosphere is extended to smaller optical depths, the growth rates change in a systematic way. The curve is compressed and lift, shifting the largest growth rates to smaller frequencies. Moreover, due to the general increase of the growth rates, some high order modes which are found to be stable in the first model, become unstable in the model with an extended atmosphere.

We emphasize that far from the edges of the TIS, the results are rather robust, in the sense that high frequency modes are excited under all conditions explored. The exact frequency of the modes and, to a less extent, the number of modes excited is, however, influenced by the details of the model. Consequently, the fact that near the edges the energy balance for some high frequency modes results in predicted growth rates that are close to zero, implies that the uncertainty in the minimum optical depth can change the position of the edges of the TIS.

\subsection{Direct effects of the magnetic field - interaction with pulsation}

Oscillations of the kind observed in roAp stars are expected to perturb the magnetic field. The magnetic field will thus react back, influencing the oscillations.

Assuming the observed oscillations are global acoustic waves deep in the interior of the star, then, in the region where the magnetic pressure and the gas pressure are comparable, they must have a magnetoacoustic nature. As the wave propagates down to the region where the gas pressure dominates, it decouples into an (essentially) acoustic and a (essentially) magnetic wave. If, as suggested by Roberts \& Soward (1983), the magnetic wave is assumed to dissipate as it propagates towards the interior of the star, due to the rapid increase in its wavenumber, the acoustic wave is constantly losing energy through the coupling with the magnetic field in the surface layers.

While studying linear, adiabatic oscillations in the presence of a magnetic field, Cunha \& Gough (2000) found that the relative amount of energy lost through the coupling 
mentioned above, changes in a cyclic way with the frequency of the modes. Moreover, according to their results, at some frequencies the associated negative growth rates can be comparable to the positive growth rates obtained by BCDGV for the same high frequency oscillations. This means that the direct effect of the magnetic field on the energy balance that determines whether a given mode is excited, can compete with the nonadiabatic effects, and must be taken into account in a consistent way. Therefore, linear, nonadiabatic calculations which include the direct effect of the magnetic field are needed, to improve our understanding of the excitation mechanism in roAp stars. Saio (private communication) mentioned that preliminary results indicate that the coupling mentioned above can also contribute to stabilizing low order modes in roAp stars.

\subsection{Wave reflection in the surface layers}

The amount of energy lost in the surface layers of the star through wave transmission, and consequent dissipation, is also an essential ingredient of the energy balance that determines whether a given mode is excited in a given roAp star. Unfortunately, the models used so far to study wave reflection in the surface layers of roAp stars did not include a magnetic field. Models used for studying the magnetic field effect on pulsations, on the other hand, tend to use boundary conditions for mode reflection/transmission which are rather artificial. The understanding of the mechanism by which waves are reflected is thus one of our present research priorities.

\section{Conclusions}

The excitation of oscillations results from a subtle balance between energy losses and gains. Thus, the study of all possible energy 'sources' and 'sinks' is absolutely necessary, if the problem of the excitation mechanism in roAp stars is to be fully understood.

In this paper we have discussed different effects that we believe will determine the excitation of the high order modes observed in roAp stars, as well as to the damping of the low order modes which, so far, have never been found. We have discussed some of the uncertainties involved in the calculations and, last, but certainly not least, we have argued that we still have a lot of work in front of us, before we can claim that the question 'why do these stars pulsate in the way they do?' has been fully answered.

\section{Acknowledgements}

MC is supported by FCT-Portugal, through the grants BPD/8338/2002 and POCTI/ FNU/43658/2001.

\section{References}

Balmforth, N.J., Cunha, M.S., Dolez, N., Gough, D.O. \& Vauclair, S., 2000, MNRAS, 323, 362 (BCDGV)

Cunha, M.S., 1999, PhD thesis, University of Cambridge, UK

Cunha, M.S., 2002, MNRAS, 333, 47

Cunha, M.S. \& Gough, D. O., 2000, MNRAS, 319, 1020

Dolez, N. \& Gough, D. O., 1982, in: J. P. Cox \& C.J. Hansen (eds.), Pulsations in Classical and Cataclysmic Variable stars, (JILA, Bouder, CO), p. 248

Dziembowski, W.A. \& Goode, P.R., 1996, ApJ, 458, 338

Gough, D. O. \& Tayler, R.J., 1966, MNRAS, 133,85

Kurtz, D. W., 1982, MNRAS, 200, 807

Moss, D.L. \& Tayler, R.J., 1969, MNRAS, 145, 217

Roberts, P. H. \& Soward, A., 1983, MNRAS, 205, 1171 


\section{Discussion}

R. TRAmpedach: Which convection theory do you use to address the interaction between convection and p-modes?

M. S. CunHA: In the models in which convection was not suppressed, we have used a nonlocal generalization of a time-dependent, mixing-length theory (Gough, 1977, ApJ 214, 196, Balmforth, 1992, MNRAS 255, 603).

R. Trampedach: In that case I would like to point out that the Gough et al. convection model suffers from the same unphysical large gradient of the turbulent pressure at the top of the convective zone as do MLT models. This results in a sharp feature in the gas pressure and temperature. I do not know how this affects the modes and their interactions with convection, but the effect is most likely significant.

T. RyABChikova: Maybe abundance gradients of metals, observed and calculated in roAp stars below $\log \tau=-1$ influence the proposed pulsation excitation mechanism?

M. S. CunhA: I believe they will have no direct effect in the excitation, because of their low abundances, when compared with hydrogen. They might have a small indirect effect, if they have a strong impact in the structure of the atmosphere. 